\title{
Managing neurocysticercosis: challenges and solutions
}

This article was published in the following Dove Press journal:

International Journal of General Medicine

16 October 2015

Number of times this article has been viewed

\section{Yannick Fogoum Fogang \\ Abdoul Aziz Savadogo \\ Massaman Camara \\ Dènahin Hinnoutondji Toffa \\ Anna Basse \\ Adjaratou Djeynabou Sow \\ Mouhamadou Mansour \\ Ndiaye}

Neurology Department, Fann Teaching Hospital, Cheikh Anta Diop University, Dakar, Senegal

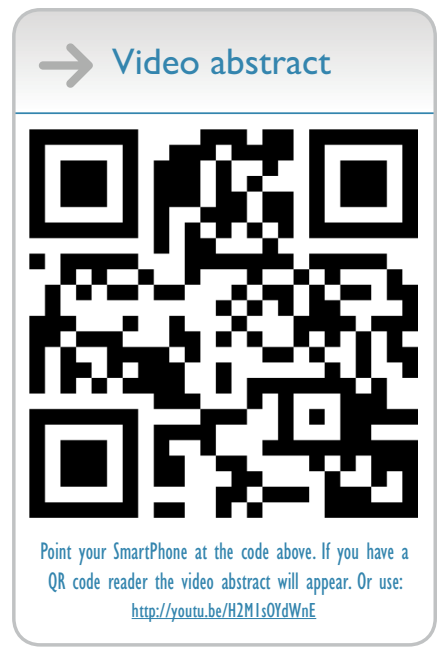

Correspondence: Yannick Fogoum Fogang Neurology Department, Fann Teaching Hospital, Cheikh Anta Diop University, PO Box 5035, Dakar, Senegal

Tel +22I 777390619

Email yanfogang@yahoo.fr
Abstract: Taenia solium neurocysticercosis (NCC) is a major cause of neurological morbidity in the world. Variability in the neuropathology and clinical presentation of NCC often make it difficult to diagnose and manage. Diagnosis of NCC can be challenging especially in endemic and resource-limited countries where laboratory and imaging techniques are often lacking. NCC management can also be challenging as current treatment options are limited and involve symptomatic agents, antiparasitic agents, or surgery. Although antiparasitic treatment probably reduces the number of active lesions and long-term seizure frequency, its efficacy is limited and strategies to improve treatment regimens are warranted. Treatment decisions should be individualized in relation to the type of NCC. Initial measures should focus on symptomatic management, with antiparasitic therapy only to be considered later on, when appropriate. Symptomatic treatment remains the cornerstone in NCC management which should not only focuses on epilepsy, but also on other manifestations that cause considerable burden (recurrent headaches, cognitive decline). Accurate patients' categorization, better antiparasitic regimens, and definition of new clinical outcomes for trials on NCC could improve management quality and prognosis of NCC. Prevention strategies targeting tapeworm carriers and infected pigs are yielding good results in local models. If local elimination of transmission is confirmed and replicated, this will open the door to cysticercosis eradication efforts worldwide.

Keywords: neurocysticercosis, Taenia solium, epilepsy, headache, albendazole, praziquantel

\section{Introduction}

Cysticercosis, an infection caused by the larval form of Taenia solium, is the most common helminthic disease of the nervous system in humans. ${ }^{1}$ It is widely prevalent in developing countries in sub-Saharan Africa, Asia, and Latin America. Cysticercosis is considered by the World Health Organization to be the most common preventable cause of epilepsy in the developing world, with an estimated 2 million people having epilepsy caused by $T$. solium infection. ${ }^{1}$ Symptomatic neurocysticercosis (NCC) accounts for approximately one-third of seizure disorders ${ }^{1-5}$ and contributes to other neurological disease, ${ }^{6}$ among which recurrent or chronic headaches are probably underestimated. $^{7}$

Neurocysticercosis is also found in industrialized countries and contributes, sometimes considerably, to the burden of disease in patients with seizures or intracranial hypertension attending emergency rooms or accessing neurological or neurosurgical departments. ${ }^{2,3}$

T. solium infection is not endemic in the USA. Therefore, NCC cases are mainly due to immigration from endemic countries rather than local transmission. In the past 
few decades, better understanding of the T. solium life cycle and modes of transmission, combined with novel diagnostic and therapeutic strategies has improved the prognosis of patients infected with $T$. solium. Despite these advances, NCC remains a major public health problem in most of the world. Millions of individuals are estimated to be infected, many of whom become symptomatic at some point in their lives. ${ }^{5-7}$ Neurocysticercosis accounts for about 50,000 deaths per year. ${ }^{8}$ Without aggressive management, which is not always available in many endemic areas, extraparenchymal NCC is still associated with high mortality rates, mainly due to intracranial hypertension, ${ }^{9,10}$ whereas mortality in parenchymal neurocysticercosis is limited to epilepsy-related deaths or a high burden of cysts. ${ }^{11}$

In the first part of this paper, we will review epidemiology, T. solium life cycle, and clinical, laboratory, and neuroimaging presentation of $\mathrm{NCC}$, which are important points to understand the principles of NCC management. We will also focus on challenges faced during NCC management, especially in low- and middle-income countries, with solutions and perspectives to overcome them.

\section{Epidemiology of neurocysticercosis Seroprevalence of Taenia solium cysticercosis}

Estimation of the prevalence of NCC in the general population is challenging because it is difficult to perform neuroimaging in a large population. ${ }^{12}$ Moreover, poor record keeping in several developing countries and socioeconomic issues skewing those who actually have relatively better access to medical care are other significant factors limiting accuracy of NCC prevalence estimate.

However, the prevalence of T. solium cysticercosis in the general population has been evaluated using a blood test.

In Latin America, the prevalence of $T$. solium cysticercosis as measured by antibody-enzyme-linked immunosorbent assay (ELISA) and/or Western blot ranged from 3.7\% to $24 \% .{ }^{13-16}$ In Asia, seroprevalence data are scarce, but reports are slowly emerging showing $T$. solium cysticercosis seroprevalence rates of $2 \%-13 \%{ }^{17,18}$

In sub-Saharan Africa, community-based seroprevalence data on T. solium cysticercosis are emerging. In cysticercosis endemic areas, seroprevalence, measured with an antigenELISA, varies from $6 \%$ to $22 \% .{ }^{19-22}$

\section{Prevalence of neurocysticercosis}

NCC is asymptomatic in approximately $50 \%$ of cases. ${ }^{7}$ Reports on autopsy results mainly come from Latin America and show NCC in $2.5 \%-6 \%$ of the examined cases. ${ }^{14}$ As autopsy results are not routinely available, indirect hospitalor community-based approaches are used in order to get an impression about the prevalence of people with NCC in certain populations. And often people suffering from epileptic seizures/epilepsy or other neurological disorders, like recurrent or chronic headaches, are included into these studies.

A recent meta-analysis of epilepsy and NCC in Latin America revealed a median NCC proportion among persons with epilepsy of $32.3 \%{ }^{23}$ Similar rates are found in other endemic regions.

A recent meta-analysis that only included African studies showed a significant association between epilepsy and cysticercosis, with an odds ratio of $3.4 .^{24}$ Prevalence rates of 144/1,000 for NCC were reported in rural settings in Ecuador. ${ }^{25}$ The total number of all people suffering from NCC, including symptomatic and asymptomatic cases, is estimated somewhere between 2.56 and 8.30 million, ${ }^{25}$ based on the range of epilepsy prevalence data available, which is between 4 and 13/1,000 for sub-Saharan Africa. ${ }^{26,27}$ This amount could be higher if the occurrence of other NCC symptoms like headache or psychiatric manifestations were not underestimated. ${ }^{7}$

In nonendemic areas, the prevalence of NCC is $0.2-0.6$ per 100,000 inhabitants in some western states of the USA, and it is diagnosed in more than $2 \%$ of patients attending emergency rooms because of seizures. ${ }^{4}$ Neurocysticercosis cases reported in western countries a few decades ago were seldom. ${ }^{28,29}$ However, a growing number of cases are registered due to the increasing flow of immigrants from endemic countries. ${ }^{28,29}$ Meanwhile, neurocysticercosis prevalence is decreasing in developing countries, both in urban and rural settings. ${ }^{30}$ Many factors may have contributed to this drop in the number of NCC cases in endemic countries, including improved sanitation, widespread use of antihelminthic drugs, and increased awareness in the general population. ${ }^{30,31}$

\section{Parasite life cycle}

Neurocysticercosis is caused by cysticercus cellulosae, the larval stage of the tapeworm T. solium (Figure 1). In the life cycle of $T$. solium, humans and pigs are the two hosts. Humans are the definitive hosts harboring adult parasites in their small intestine in the usual cycle of transmission. Eggs produced by adult worms are passed with feces of Taenia carriers. In areas with poor orofecal hygiene, free-roaming pigs can get access to human feces and ingest infective eggs. When those eggs are ingested by pigs, they enter the 


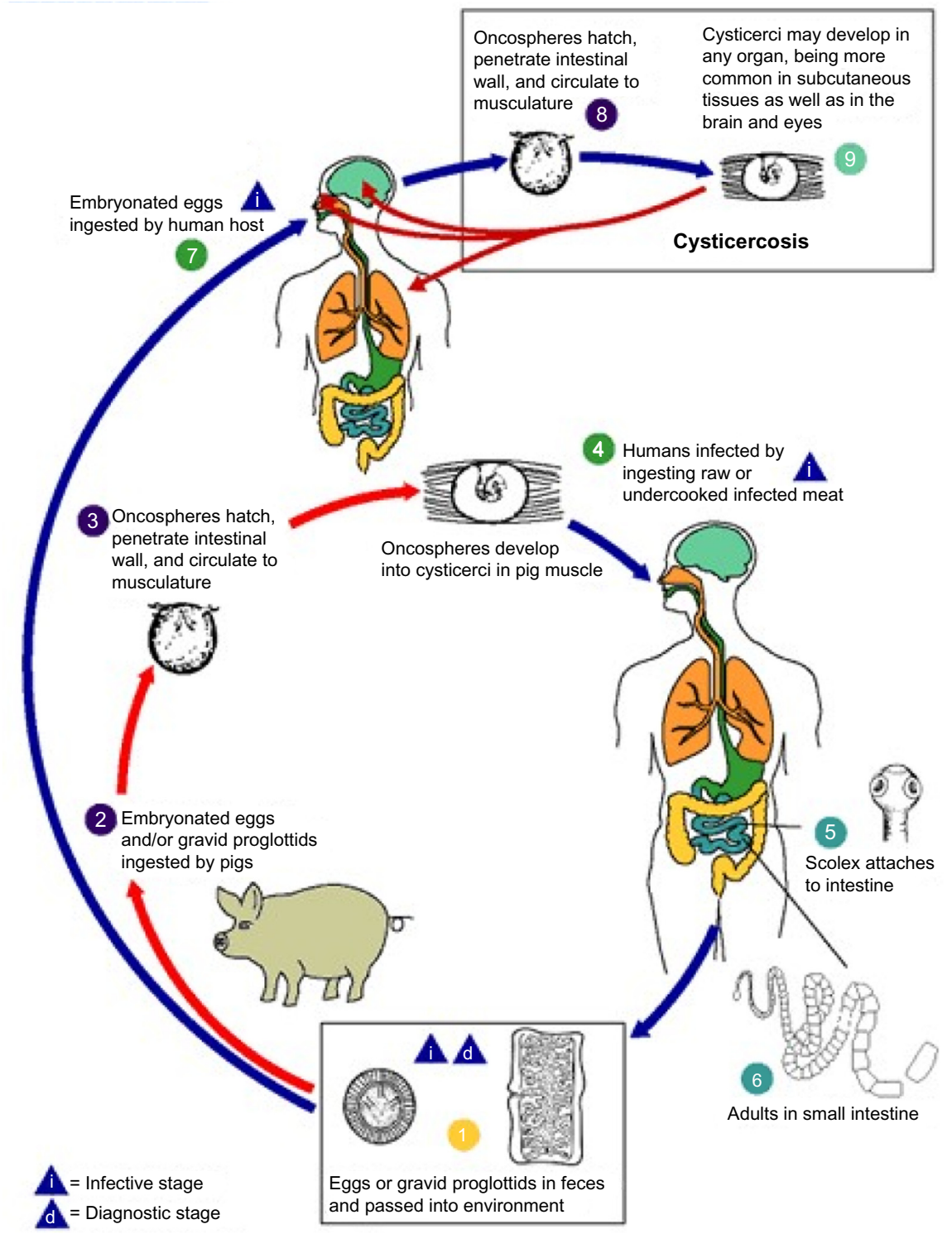

Figure I Life cycle of Taenia solium cysticerci.

Note: Reproduced from Centers for Disease Control and Prevention. Cysticercosis. Atlanta, GA: Centers for disease control and prevention. Available from: http://www. cdc.gov/parasites/cysticercosis/biology.html. ${ }^{105}$

bloodstream after crossing the intestinal wall and mature into oncospheres and then into metacestodes which are found in usual intermediate host tissues, especially striated muscles. Metacestodes then evolve into cysticerci, which are vesicles containing an invaginated scolex. These larvae evaginate in the gastrointestinal tract of humans consuming undercooked pork infected with cysticerci, adhere to the mucosa, and begin to grow, forming adult worms. When those adult worms become gravid and produce eggs, which are excreted with human feces, the life cycle is completed. The only source of infection for humans and pigs is the human tapeworm carrier. Moreover, the adult $T$. solium tapeworm has a very high biotic potential, meaning that a single worm can infect many hosts in its surroundings. For all these reasons, the tapeworm carrier is the main target of control interventions. Humans can also be intermediate host after ingestion of eggs through interpersonal transmission (direct contact or food handling). This explains the occurrence of NCC in persons neighboring of individuals immigrating from endemic areas and infested by $T$. solium. . $^{5,32}$

\section{Neuropathology of neurocysticercosis}

When cysticerci enter the central nervous system, they are in a vesicular stage, with the parasite harboring an invaginated scolex surrounded by a translucid vesicular fluid and recovered by a transparent membrane (Figure 2). Cysticerci may remain in this state for years, or may degenerate after 

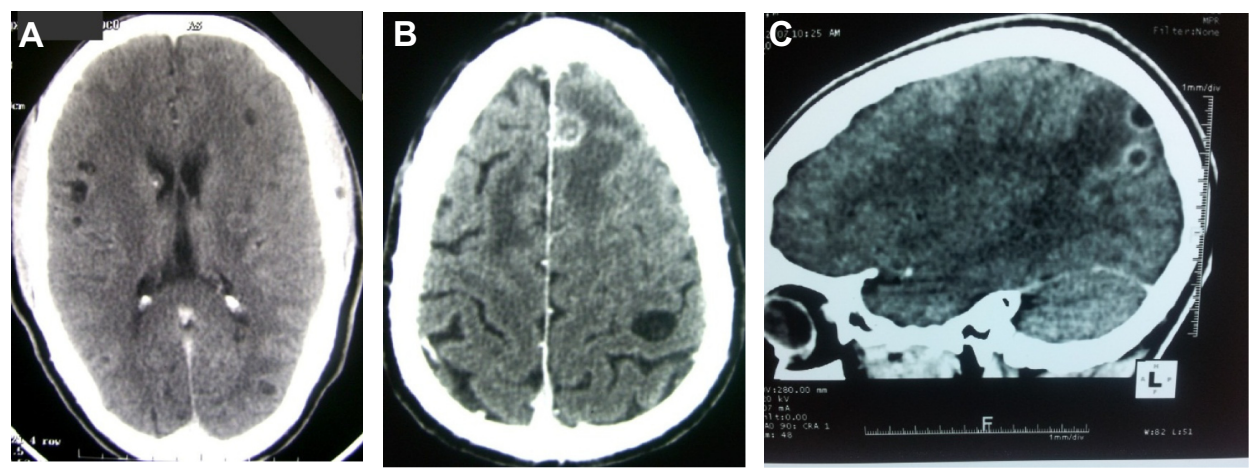

Figure 2 CT imaging of human neurocysticercosis.

Notes: Viable cysts on brain CT (A); lesions at different stages of involution, granuloma, and viable cyst (B). Enhancing granuloma with perilesional edema (C). (C) (C) 20I4 Fogang et al.; licensee BioMed Central Ltd. Reproduced from Fogang YF et al. Cerebral neurocysticercosis mimicking or comorbid with episodic migraine? BMC Neurol. 20I4; 14:138. Creative Commons license and disclaimer available from: http://creativecommons.org/licenses/by/4.0/legalcode. ${ }^{40}$

Abbreviation: CT, computed tomography.

host immunologic response. Colloidal stage is the first stage of involution of cysticerci and is characterized by a turbid vesicular fluid with hyaline degeneration of the scolex. In the next stage, the cyst wall thickens and the scolex is mineralized. At this level, which is called the granular stage, the cysticercus is no longer viable (Figure 2). The final stage of this degenerative process is the calcification of parasite remnants. It is not uncommon to find cysticerci in different degenerative stages in the same person (Figure 2). A peculiar neuropathological pattern of NCC is the racemose form in which parasites are made of several membranes clustering with no identifiable scolex. The racemose form is usually observed in cerebrospinal fluid (CSF) cisterns and may attain a large size. ${ }^{5}$

\section{Clinical manifestations}

Clinical manifestations of NCC can vary from completely asymptomatic infection to severe disease and death. Signs and symptoms of NCC are usually nonspecific, with the exception of ocular cysts, which can be diagnostic. In endemic areas, NCC is regarded as the great imitator because it can mimic almost any neurological disorder. ${ }^{33}$ Disease severity and clinical manifestations are indicative of the characteristics of infection (number, size, and location of cysts, and intensity of the host's immune response). The major determinant of the characteristics of symptomatic NCC is whether the parasites are located in the brain parenchyma or in the extraparenchymal spaces. ${ }^{34}$

\section{Parenchymal neurocysticercosis Seizures and epilepsy}

Epilepsy is the most frequently reported symptom in NCC. In a systematic review, epileptic seizures were reported in up to $80 \%$ of patients with symptomatic NCC. ${ }^{35}$ Seizures are more frequently generalized in NCC, although many of these seizures may correspond to secondary generalization. ${ }^{35}$ Epileptic seizures mainly occur in two settings, either as acute symptomatic seizures in the context of active cysts or granuloma formation or as "chronic epilepsy", associated with end-stage calcified lesions..$^{36,37}$

\section{Headaches}

Symptomatic adults may complain of headaches in $27.7 \%$ of cases ${ }^{35}$ although this may be an underestimation. A headache in some patients may indicate raised intracranial pressure. ${ }^{38}$ Often, a headache in people with NCC is recurrent or chronic, and presents a tension-type like headache or migraine. ${ }^{25,39,40}$ Accumulating evidence from numerous case reports and epidemiologic studies has established an association between NCC and migraine-like headaches, with an odds ratio between 2.65 and $3.39,,^{40-42}$ similar to that for epilepsy. Given that recurrent headaches are more prevalent than epilepsy even in regions where NCC is endemic, more attention from practitioners and researchers should be given to the association of NCC with recurrent headaches, as it is established that frequent and chronic headaches are associated with an altered quality of life and considerable socioeconomic burden. However, there is no sufficient evidence to recommend systematic laboratory tests or neuroimaging for NCC in patients presenting with recurrent or chronic headaches, even in endemic areas.

\section{Other manifestations}

Adults with NCC can also present focal neurological signs (11.8\%), signs of raised intracranial pressure (16.3\%), meningeal signs $(5.6 \%)$, gait abnormalities $(5.6 \%)$, and altered mental state/psychiatric symptoms (28.1\%), among others. $^{35,43,44}$ 


\section{Extraparenchymal neurocysticercosis}

In extraparenchymal NCC, cysticerci may lodge in the ventricular system or subarachnoid space and a potentially lifethreatening acute intracranial hypertension may develop. CSF flow obstruction most frequently occurs when cysts lodge in the fourth ventricle and progressively causes hydrocephalus. Nash and $\mathrm{Garcia}^{45}$ in their review subdivided extraparenchymal NCC into four subgroups, consisting of ventricular NCC, subarachnoid NCC of the brain convexity, subarachnoid NCC of the lateral fissure, and basal subarachnoid NCC owing to different locations, pathologies, and ensuing clinical characteristics.

\section{Other forms of neurocysticercosis}

Cysticercal encephalitis, which symptons can present with intracranial hypertension, is related to a massive parenchymal infection inducing an intense immune response with diffuse brain edema. ${ }^{46-48}$ Spinal cord involvement is rare and accounts for $1 \%-5 \%$ of all NCC cases. ${ }^{7}$

\section{Diagnosis}

Histological confirmation of the diagnosis of NCC is not possible in the majority of cases. Thus, diagnosis is usually based on neuroimaging and immunological tests. Despite modern neuroimaging methods and reliable immune diagnostic tests, diagnosis of neurocysticercosis can still be a challenge. This is mostly explained by the poor specificity of clinical and neuroimaging findings, and suboptimum predictive values in immunodiagnostic tests, particularly in endemic settings.

\section{Neuroimaging}

Neuroimaging is the method of choice for the diagnosis of NCC, determination of need for medical or surgical treatment, and assessment of effectiveness of interventions. ${ }^{36,43} \mathrm{It}$ includes cranial computed tomography (CT) and/or magnetic resonance imaging (MRI), which should be combined with serological tests. In neuroimaging, only a lesion showing the scolex (head of the parasite) is classified as a definite NCC lesion and considered pathognomonic. ${ }^{38,49-51}$ Active NCC is defined as the presence of any cystic lesions (with or without scolex) or lesions with ring enhancement. Nodular enhancing lesions are often termed transitional. In contrast, parenchymal calcifications are classified as inactive. ${ }^{52,53}$ Lesions highly suggestive of NCC are of the active, transitional, and inactive type..$^{52,53}$ The latter should only be included if calcifications are solid, dense, and multiple and located supratentorially, measuring 1-10 $\mathrm{mm}$ in diameter in the absence of any other disease that may explain the calcifications. ${ }^{52}$ In addition, cranial lesions in different evolutionary stages further support the diagnosis of NCC. ${ }^{36}$

Beyond the limited accessibility of neuroimaging methods in developing countries, diagnosis of NCC on neuroimaging is often challenging even in developed countries. For example, thin-walled cysts are often missed by CT and even MRI if dedicated imaging is not performed. Additionally, NCC can frequently be missed if the radiologist evaluating the imaging is not a neuroradiologist. Where available, optimal neuroimaging protocols should be used when NCC is suspected to increase the diagnostic yield. In this line, fast imaging employing steady-state acquisition (FIESTA) can be very helpful. It is a higher speed, better resolution, thinner cut way to see the thin walls of the cyst which can often be missed by traditional MRI techniques and nonneuroradiologists. FIESTA should be asked for by the ordering provider when the radiologist protocols the study.

\section{Immunological diagnosis}

As neuroimaging is often unavailable in sub-Saharan Africa, serological results are important for guidance as to which patient should be referred to an imaging center. The best documented serological test is the enzyme-linked immunoelectrotransfer blot (EITB) assay, which uses lentil lectin purified glycoprotein antigens to detect antibodies to T. solium in serum. EITB specificity approaches $100 \%$ with a sensitivity around $98 \%$ for patients with two or more live parasites in the nervous system. Thus, people with more than one viable cyst or subarachnoid disease at the time of testing will have a positive serology. EITB does not cross-react with heterologous infections. ${ }^{54}$ A negative serology in patients should lead to the investigation of alternative diagnoses. The sensitivity of antibody detection by EITB seems to be slightly lower in CSF than in serum ( $90 \%$ vs $100 \%) .{ }^{55}$ A major weakness of EITB is its low sensitivity $(50 \%-60 \%)$ in patients with one intracranial cysticercus. Thus, EITB is frequently falsely negative in patients with a single intracranial cysticercus (including the Indian variant of solitary cerebral cysticercus granuloma) or in those with calcified cysticerci. ${ }^{36,54}$ Therefore, a negative test cannot exclude neurocysticercosis.

However, antibodies to $T$. solium are frequently reported in the asymptomatic general population in endemic regions; their presence can suggest exposure to the parasite or current or past asymptomatic infections. Since antibody assays show cysticercus infection, the serum of any patient with muscular or subcutaneous cysticercosis, but no brain involvement, might test positive, thereby lowering the specificity of these assays to diagnose NCC. 


\section{CSF analysis}

When lumbar puncture is not contraindicated, CSF analysis offers additional information to exclude other potentially life-threatening infections when imaging is not typical of NCC, especially in endemic regions where asymptomatic cysticercosis is common. Important differential diagnoses in NCC-endemic regions mainly include cerebral malaria, bacterial or tuberculous meningitis, and encephalitis. Unlike bacterial meningitis, findings in tuberculous meningitis and viral encephalitis may resemble those in NCC. However, in patients with intraparenchymal NCC, CSF analysis is frequently unremarkable or may show moderate and nonspecific abnormalities. ${ }^{?}$

\section{Diagnostic criteria}

The poor specificity of clinical and neuroimaging findings, and suboptimum predictive values of immunodiagnostic tests particularly in endemic settings have favored the development of diagnostic criteria for NCC. Diagnostic criteria for NCC were published, which used objective clinical, radiological, immunological, and epidemiological data. ${ }^{56}$ These criteria are classified on the basis of their diagnostic strength as absolute, major, minor, or epidemiologic. They help to establish the degree of certainty of NCC diagnosis (Table 1). Although not systematically validated because of the absence of a comparative gold standard, these criteria have become highly useful in the diagnosis of NCC. However, some authors have raised concerns about their validity and applicability. ${ }^{57}$ Newly developed immunodiagnostic tests and imaging techniques might help to improve these criteria in the near future.

\section{Management of neurocysticercosis}

Before therapy is planned, proper characterization of the specific type and brain involvement of NCC is important. ${ }^{58}$ Therapeutic approaches might include symptomatic therapy, antiparasitic treatment, or surgery. Often, more than one of these options are needed. Preventive measures are also important for the control and, eventually, eradication of NCC. Currently, there are no standard treatment guidelines for NCC, although suggestions have been made. ${ }^{36,38,43-46,48,53,59-64}$ Therefore, treatment has to be tailored to the individual case.

\section{Symptomatic treatment}

Symptomatic treatment is the mainstay of NCC management and should not be delayed. It involves antiepileptic drugs (AEDs), anti-inflammatories, analgesics, and management of intracranial hypertension when present. ${ }^{53}$
Table I Diagnostic criteria for neurocysticercosis ${ }^{94}$

\section{Diagnostic criteria}

Absolute criteria

- Histologic demonstration of the parasite from biopsy of a brain or spinal cord lesion

- Evidence of cystic lesions showing the scolex on neuroimaging studies

- Direct visualization of subretinal parasites by funduscopic examination Major criteria

- Evidence of lesions highly suggestive of neurocysticercosis on neuroimaging studies

- Positive serum immunoblot for the detection of anticysticercal antibodies

- Resolution of intracranial cystic lesions after therapy with albendazole or praziquantel

- Spontaneous resolution of small single-enhancing lesions

Minor criteria

- Evidence of lesions suggestive of neurocysticercosis on neuroimaging studies

- Presence of clinical manifestations suggestive of neurocysticercosis

- Positive CSF ELISA for detection of anticysticercal antibodies or cysticercal antigens

- Evidence of cysticercosis outside the CNS

Epidemiologic criteria

- Individuals coming from or living in an area where cysticercosis is endemic

- History of frequent travel to disease-endemic areas

- Evidence of a household contact with Taenia solium infection

Degrees of diagnostic certainty

Definitive diagnosis

- Presence of one absolute criterion

- Presence of two major plus one minor or one epidemiologic criteria Probable diagnosis

- Presence of one major plus two minor criteria

- Presence of one major plus one minor and one epidemiologic criteria - Presence of three minor plus one epidemiologic criteria

Note: Copyright $\odot$ Gabriël et al. Reproduced from Gabriël S, Blocher J, Dorny P, et al. Added value of antigen ELISA in the diagnosis of neurocysticercosis in resource poor settings. PLoS Negl Trop Dis. 2012;6:el851. ${ }^{94}$ Creative Commons license and disclaimer available from: http://creativecommons.org/licenses/by/4.0/legalcode. Abbreviations: CSF, cerebrospinal fluid; ELISA, enzyme-linked immunosorbent assay; CNS, central nervous system.

\section{Treatment of epileptic seizures or epilepsy}

Recurrent seizures are most often the main or sole manifestation of parenchymal brain cysticercosis. In most endemic countries, NCC occurs in about $30 \%$ of patients with epilepsy or with seizures. ${ }^{6,30,51,65}$ Thus, appropriate management of seizures or epilepsy in NCC is crucial to alleviate the burden of this disease. In many countries where NCC is endemic, AEDs (phenobarbitone, phenytoin, and occasionally carbamazepine) are relatively easy to access. Single first-line therapy using one of the three available AEDs usually enables adequate seizure control in people with NCC, although the effect has only been demonstrated with phenobarbitone and carbamazepine ${ }^{66}$ Use of AEDs in NCC does not differ 
from use in other similar types of epilepsy and seizures. ${ }^{43}$ A recent study comparing responses to AEDs in people with epilepsy and calcified NCC, those with other structural cerebral lesions, and those without obvious cerebral lesions has shown no difference in treatment response in the three groups, which may indicate that free access to and choice of all available AEDs does not improve seizure outcome in people with $\mathrm{NCC}^{44}$

\section{Treatment with anti-inflammatory drugs}

In ventricular, subarachnoid, and encephalitic (multiple intraparenchymal cysts with edema development) NCC, one has to consider a reactive increase of intracranial pressure that may be caused by inflammation due to cyst degeneration either naturally or with the use of antiparasitic medication. For this reason, in these complicated conditions, outpatient treatment has to be discouraged since (although rare) decompensation of intracranial hypertension has been reported. ${ }^{67,68}$ However, the occurrence of this potentially life-threatening complication can be minimized by the simultaneous use of steroids in ventricular and subarachnoid forms and administration of steroids only in patients with massive infection. ${ }^{46,64}$ In patients with a low-to-medium amount of intraparenchymal cysts who do not show severe manifestations of intracranial hypertension, steroids should be administered during the first days of antiparasitic drugs and maintained until the main symptoms have resolved (eg, chronic progressive headache, acute symptomatic epileptic seizures). Although the use of steroids for controlling inflammation has been well demonstrated, it must be mentioned that due to their immunosuppressive properties, their use could be involved in the nonresponse to cysticidal drugs in patients with extraparenchymal NCC. ${ }^{69}$ Studies evaluating this aspect are needed. There has been so far no standardized steroid doses in the treatment of NCC..$^{37,70,71}$ In single-enhancing granulomatous lesions, the use of steroids is associated with a decrease in epileptic seizures and a significant clearance of cysts on CT. ${ }^{37,46,72-75}$ Although the use of steroids for the treatment of NCC shows clear beneficial effects, one should keep in mind that abrupt withdrawal may lead to rebound neurological symptoms/signs. Thus, steroids need to be tapered off slowly. ${ }^{43}$

For patients who develop recurrent cerebral inflammation or serious side effects attributed to steroids, methotrexate may be useful. ${ }^{76}$

\section{Treatment of headaches}

A headache is the second more encountered manifestation of NCC. This frequency is probably underestimated as a headache is not systematically assessed in studies dealing with NCC. A headache in the setting of raised intracranial pressure will be alleviated by administration of steroids, osmotic diuretics, and/or surgery depending on the mechanism involved in raised intracranial pressure. In patients presenting recurrent or chronic migraine-like headaches, analgesics or nonsteroidal anti-inflammatory drugs can be prescribed for attacks ${ }^{40}$ but caution should be taken to avoid gastric ulcer and medication overuse, which can worsen headaches. Patients with frequent headaches can be prescribed preventive headache medications. When headaches are associated with epilepsy, priority should be given to medications effective on both conditions like sodium valproate or topiramate when available. However, clinical trials on treatment of recurrent or chronic headaches associated or attributed to NCC are warranted to objectively define treatment recommendations.

\section{Management of intracranial hypertension}

Intracranial hypertension can be caused by cysticercotic arachnoiditis, mass effect of cysts located in basal subarachnoidal cisterns, or the obstruction of CSF pathway by ventricular cysts. Resolution of intracranial hypertension is crucial and urgent. High-dose steroids, osmotic diuretics, and surgery will be used when indicated.

\section{Antiparasitic treatment}

Antiparasitic therapy in NCC is not an urgent intervention and should be initiated only when the patient is stable without symptoms. ${ }^{7}$ Two main antiparasitic agents are commonly used: albendazole and praziquantel. The goal of antiparasitic therapy can be defined as the destruction of cysts with concurrent control of the host immune response using anti-inflammatory medication such as corticosteroids. This strategy has been used worldwide and prevents the prolonged inflammation related to cyst degeneration. It therefore improves clinical evolution and outcome. ${ }^{48}$ The existing NCC regimes have a partial efficacy. One course of antiparasitic treatment yields a $60 \%-70 \%$ cysts resolution versus $40 \%$ in untreated patients. ${ }^{77-79}$ Unfortunately, only 30\%-40\% of patients are free of viable brain cysts. ${ }^{77-79}$ Antiparasitic drugs are recommended only for active disease. Dosages and the duration of treatment vary according to the number, size, location, and developmental stage of the cysts; surrounding inflammatory edema; clinical symptoms or signs; as well as potential risk factors of treatment.

It is critical to note that the administration of antiparasitic drugs may induce or increase preexisting cerebral edema. 
Thus, antiparasitic treatment is contraindicated in intracranial hypertension, subarachnoid NCC, and NCC encephalitis. ${ }^{43}$ In these conditions, antiparasitic drugs should be delayed, and steroids administered first. Efficacy and safety of antiparasitic treatment for intraparenchymal NCC have recently been addressed in a meta-analysis. ${ }^{80}$ Albendazole therapy administered with or without corticosteroids is well tolerated and seems to decrease both long-term seizure frequency and the number of cysts in NCC ${ }^{80}$ However, these authors did not find sufficient evidence to support or refute the use of praziquantel. ${ }^{80}$

\section{Albendazole}

Albendazole, compared to praziquantel, has a better penetration into the central nervous system, a greater cysticidal effect, less interaction with other drugs, and lower price. For these reasons, it is the antiparasitic agent of choice in NCC. ${ }^{36,43,45,60}$ Unlike praziquantel, albendazole has no effect on adult T. solium tapeworm. The regimen has been adapted according to worldwide clinical experience and currently a 1to 2 -week course of $15 \mathrm{mg} / \mathrm{kg}$ in two divided doses 12 hours apart has been recommended. ${ }^{45,46,59,61,64}$ A Mexican study detected considerable variation in pharmacokinetic properties and plasma concentrations of albendazole sulfoxide. Oral bioavailability of albendazole may vary with ethnicity, with potential impact on drug efficacy. ${ }^{81}$ As the clearance of cysts with both albendazole and praziquantel is not satisfactory, studies have tried higher doses of $30 \mathrm{mg} / \mathrm{kg} / \mathrm{d}$, but the safety profile so far has not been assessed. ${ }^{60}$ In subarachnoid NCC, albendazole at $30 \mathrm{mg} / \mathrm{kg} / \mathrm{d}$ during 8 days combined with corticosteroids is shown to be safe and more effective than the usual dose. ${ }^{82}$ A single cycle of high-dose albendazole however seems to be insufficient in intraventricular NCC and giant cysticerci, requiring retreatment. Some authors retreat if cysts persist on neuroimaging at 3 or 6 months. ${ }^{82}$ Also, there is no consensus on the retreatment regimen which may include the same dose of albendazole, or a combination of albendazole (15 mg/kg/d) and praziquantel (50 mg/kg/d). Treatment with albendazole has also been shown to be effective in solitary cerebral cysticercus granuloma in that it accelerated resolution of the granuloma with the possibility of early withdrawal of AEDs and also offered some protection against recurrence of seizures. ${ }^{62,79,83,84}$ However, methodological shortcomings of some of the trials have been pointed out, and it is unclear whether albendazole should be administered alone or in combination with steroids. ${ }^{62}$ An earlier study even demonstrated that treatment with antiparasitics may not be required at all. ${ }^{85}$

\section{Praziquantel}

Praziquantel is widely available in sub-Saharan Africa and displays strong activity against schistosomes and cestodes. ${ }^{48}$ The most effective regimen applies $50 \mathrm{mg} / \mathrm{kg}$ in three divided doses over 2 weeks to 30 days. Alternatively, $25 \mathrm{mg} / \mathrm{kg}$ given 2-hourly in a single-day scheme leads to a $90 \%$ reduction of the direct costs in relation to the traditional scheme as shown by studies from Latin America. ${ }^{48,59,86,87}$ The singleday course may be useful in patients with low cyst burden or single parenchymal cysts. However, it shows limitations in its use in multicystic disease, ${ }^{88}$ which unfortunately is the prevailing phenotype in most low- and middle-income countries. ${ }^{46,64}$

\section{Combined treatment with albendazole and praziquantel}

There is a relative scarcity of clinical trials assessing a potential role of combined antiparasitic therapy in NCC. Most knowledge relies on case reports. ${ }^{69,89-91}$ However, it has been demonstrated that praziquantel augments albendazole sulfoxide plasma concentrations, which may implicate treatment benefit in terms of shorter duration of treatment and therefore less time to exposure of common adverse effects. ${ }^{60}$ A trial of combination therapy in NCC using albendazole and praziquantel has recently been conducted showing an increased cysticidal effect in patients with multiple cerebral cysts without increased side effects. ${ }^{43}$

\section{Surgery}

CSF drainage or shunt placement is needed for most cases of hydrocephalus because of subarachnoid NCC, although highdose corticosteroids (dexamethasone, $16 \mathrm{mg} / \mathrm{kg} / \mathrm{d}$ or more) frequently lead to temporary control of hydrocephalus. ${ }^{43}$ Ventriculocisternostomy with third ventricle fenestration might stop the need for a shunt device, probably improving the patient's prognosis, because shunt malfunction or infection is a common cause of morbidity. ${ }^{92}$ Mortality has been related to the number of shunt revisions patients have undergone, ${ }^{92}$ but oral prednisone might reduce the risk of shunt dysfunction. ${ }^{93}$ Treatment of giant cysts in the lateral fissure is controversial. Surgical resection may be an option after failure of appropriate medical treatment. ${ }^{43}$

\section{Treatment without neuroimaging}

Neuroimaging is rarely available in NCC-endemic areas in resource-limited countries. A positive result on $T$. solium cysticercosis serology may indicate active cysticercosis. ${ }^{94}$ However, treatment with an antiparasitic agent must not 
be initiated on the basis of serology alone as cyst stage and presence of edema cannot be estimated. Blind trials with albendazole or praziquantel should be avoided when neuroimaging is not available, given the potential risk of aggravating raised intracranial pressure and brain herniation related to antiparasitic drugs. Hence, in the absence of neuroimaging, patients should be treated symptomatically only. ${ }^{46,64}$

\section{Prevention of neurocysticercosis}

Neurocysticercosis can be eradicated as it was done in European countries by the end of the 19 th century. T. solium infection is one of a few diseases targeted for focal elimination and eventual eradication. ${ }^{95}$ Advances in our knowledge of the parasite life cycle and modes of transmission have yielded better preventive strategies. To be effective, eradication strategies must be directed to all the targets, particularly human carriers of the adult tapeworm, infected pigs, and eggs in the environment. Since these targets represent steps of T. solium life cycle, inadequate control of one of them may result in a rebound in the incidence of taeniasis/cysticercosis at the end of the program. ${ }^{96}$

\section{Treatment of taeniasis}

The only source of infection for humans and pigs is the human tapeworm carrier. Moreover, the adult T. solium tapeworm has a very high biotic potential, meaning that a single worm can infect many hosts in its surroundings. For all these reasons, the tapeworm carrier is the main target of control interventions. Tapeworm carriers may be treated and cured either by case diagnosis and individualized treatment or by mass treatment of the human population with a single oral dose of either niclosamide ( $2 \mathrm{~g}$ in adults) or praziquantel $(5-10 \mathrm{mg} / \mathrm{kg})$. Praziquantel should be used with caution because there is a risk, although rare, that the administration of antiparasitic drugs to an individual who has silent neurocysticercosis could trigger seizures or other neurological symptoms. ${ }^{97}$

\section{Treatment of infected pigs}

Treatment of infected pigs would avoid future taeniasis infections and increase the likelihood of elimination. Oxfendazole, given as a single oral dose of $30 \mathrm{mg} / \mathrm{kg}$, is close to $100 \%$ effective to destroy muscle cysts; it is not expensive and has no major side effects. Death of cysts usually occurs within a few weeks. The withdrawal period of oxfendazole at this dose was calculated to be 17 days. Decrease in pig infection is more marked when pigs are treated at 9 months of age. ${ }^{98}$

\section{Pig vaccination}

Pig immunization using antigens from the scolex, the entire cyst, or from the oncosphere has demonstrated diverse degrees of protection. ${ }^{99-101}$ Improved pig vaccines were more recently developed. TSOL18 is an oncospheral recombinant protein. Two doses of TSOL18 vaccine provide almost absolute protection to further infection, ${ }^{102,103}$ a protection that was later confirmed in field trials. ${ }^{104}$

\section{Other potential targets for intervention}

Neurocysticercosis is common where there is clustering of conditions favoring the transmission of T. solium, including low level of education, poverty, deficient disposal of human feces, slaughtering of pigs without veterinary control, and presence of free-roaming pigs around households. Targeting all these factors, especially in endemic regions, is a challenging task, which should involve a synergic action at different levels, including health workers, policy makers, and general population.

\section{Conclusion and perspectives}

Neurocysticercosis is a public health problem in most developing countries and is reported with some frequency in industrialized countries due to immigration. Populations facing poverty and lack of health care facilities in endemic countries are more concerned. These conditions make accurate diagnosis of NCC very challenging in the absence of neuroimaging methods or appropriate immunologic tests. Treatment of NCC is mostly symptomatic. Assuming that epilepsy and headaches are the most common manifestations of NCC, even a symptomatic approach will face the same difficulties than that encountered in the general management of primary epilepsies or headaches (huge therapeutic gap). Available specific NCC drugs yield suboptimum results, and can be dangerous if staging, location, and number of central nervous system lesions are not previously established by neuroimaging. New drugs with better pharmacokinetic profiles and efficacy are warranted. Further research is also needed to provide evidence-based diagnostic techniques and therapies. Future studies should also focus on clinical outcomes rather than CT outcomes, as the two do not always correlate. New clinical outcomes like headaches and cognitive decline which were largely underestimated in previous clinical trials on NCC should be taken into consideration.

Neurocysticercosis can also be conceptualized as a human model for development of seizures and epilepsy, as well as for recurrent headaches or migraine. Well-designed studies should yield valuable information about genetic predisposition, pathological mechanisms, and potential therapeutic 
targets for chronic epilepsy or headaches. It is reasonable to consider that control of NCC in endemic countries would significantly impact the burden of epilepsy and headaches in these settings. Furthermore, gain of interest for this neglected disease will probably enhance (global) research on this topic which has so far been of limited interest for researchers in industrialized countries. Socioeconomic development in endemic regions remains a key aspect for cysticercosis eradication or tight control. If local elimination of transmission is confirmed and replicated, this will open the door to cysticercosis eradication efforts worldwide.

\section{Disclosure}

The authors report no conflicts of interest in this work.

\section{References}

1. Singh G, Burneo JG, Sander JW. From seizures to epilepsy and its substrates: neurocysticercosis. Epilepsia. 2013;54:783-792.

2. Ong S, Talan DA, Moran GJ, et al. Neurocysticercosis in radiographically imaged seizure patients in US emergency departments. Emerg Infect Dis. 2002;8:608-613.

3. Croker C, Redelings M, Reporter R, Sorvillo F, Mascola L, Wilkins P. The impact of neurocysticercosis in California: a review of hospitalized cases. PLoS Negl Trop Dis. 2012;6:1-6.

4. Coyle CM, Mahanty S, Zunt JR, et al. Neurocysticercosis: neglected but not forgotten. PLoS Negl Trop Dis. 2012;6(5):e1500.

5. Del Brutto OH, Garcia HH. Neurocysticercosis. Handb Clin Neurol. 2013;114:313-325.

6. Ndimubanzi PC, Carabin H, Budke CM, et al. A systematic review of the frequency of neurocyticercosis with a focus on people with epilepsy. PLoS Negl Trop Dis. 2010;4:e870.

7. Winkler AS, Richter H. Landscape analysis: management of neurocysticercosis with an emphasis on low- and middle-income countries (LMIC). Available from: http://apps.who.int/iris/ bitstream/10665/152896/1/WHO_HTM_NTD_NZD_2015.05_eng. pdf. Accessed May 4, 2015.

8. Roman G, Sotelo J, Del Brutto O, et al. A proposal to declare neurocysticercosis an international reportable disease. Bull World Health Organ. 2000;78(3):399-406.

9. DeGiorgio CM, Houston I, Oviedo S, Sorvillo F. Deaths associated with cysticercosis. Report of three cases and review of the literature. Neurosurg Focus. 2002;12:e2.

10. Fleury A, Carrillo-Mezo R, Flisser A, Sciutto E, Corona T. Subarachnoid basal neurocysticercosis: a focus on the most severe form of the disease. Expert Rev Anti Infect Ther. 2011;9:123-133.

11. Bhattarai R, Budke CM, Carabin H, et al. Estimating the nonmonetary burden of neurocysticercosis in Mexico. PLoS Negl Trop Dis. 2012;6:e1521.

12. Fleury A, Gomez T, Alvarez I, et al. High prevalence of calcified silent neurocysticercosis in a rural village of Mexico. Neuroepidemiology. 2003;22:139-145.

13. Flisser A, Sarti E, Lightowlers M, Schantz P. Neurocysticercosis: regional status, epidemiology, impact and control measures in the Americas. Acta Trop. 2003;87:43-51.

14. Murrell KD, Dorny P, Flisser S, et al. WHO/FAO/OIE Guidelines for the surveillance, prevention and control of taeniosis/cysticercosis, 2005. Paris, France: OIE. Available from: http://www.oie.int/doc/ged/d11245. pdf. Accessed November 7, 2014.

15. Flisser A, Gyorkos TW. Contribution of immunodiagnostic tests to epidemiological/intervention studies of cysticercosis/taeniosis in Mexico. Parasite Immunol. 2007;29:637-649.
16. Flisser A. Epidemiology of neurocysticercosis in Mexico: from a public health problem to its control. In: Foyaca-Sibat $\mathrm{H}$, editor. Novel Aspects on Cysticercosis and Neurocysticercosis. Rijeka, Croatia: InTech; 2013:307-340.

17. Rajshekhar V, Joshi DD, Doanh NQ, van De N, Xiaonong Z. Taenia solium taeniosis/cysticercosis in Asia: epidemiology, impact and issues. Acta Trop. 2003;87:53-60.

18. Li T, Craig PS, Ito A, et al. Taeniasis/cysticercosis in a Tibetan population in Sichuan Province, China. Acta Trop. 2006;100:223-231.

19. Carabin H, Millogo A, Praet N, et al. Seroprevalence to the antigens of Taenia solium cysticercosis among residents in three villages in Burkina Faso: a cross-sectional study. PLoS Negl Trop Dis. 2009;3:e555.

20. Kanobana K, Praet N, Kabwe C, et al. High prevalence of Taenia solium cysticercosis in a village community of Bas-Congo, Democratic Republic of Congo. Int J Parasitol. 2011;41:1015-1018.

21. Mwape KE, Phiri IK, Praet N, et al. Taenia solium infections in a rural area of Eastern Zambia-a community based study. PLoS Negl Trop Dis. 2012;6:e1594.

22. Mwanjali G, Kihamia C, Kakoko DV, et al. Prevalence and risk factors associated with human Taenia solium infections in Mbozi District, Mbeya Region, Tanzania. PLoS Negl Trop Dis. 2013;7:e2102.

23. Bruno E, Bartoloni A, Zammarchi L, et al. COHEMI Project Study Group. Epilepsy and neurocysticercosis in Latin America: a systematic review and meta-analysis. PLoS Negl Trop Dis. 2013;7(10): e2480.

24. Quet F, Guerchet M, Pion SDS, Ngoungou EB, Nicoletti A, Preux PM. Meta-analysis of the association between cysticercosis and epilepsy in Africa. Epilepsia. 2010;51:830-837.

25. Cruz ME, Schantz PM, Cruz I, et al. Epilepsy and neurocysticercosis in an Andean community. Int J Epidemiol. 1999;28:799-803.

26. Edwards T, Scott AG, Munyoki G, et al. Active convulsive epilepsy in a rural district of Kenya: a study of prevalence and possible risk factors. Lancet Neurol. 2008;7:50-56.

27. Winkler AS, Kerschbaumsteiner K, Stelzhammer B, Meindl M, Kaaya J, Schmutzhard E. Prevalence, incidence and clinical characteristics of epilepsy - a community-based door-to-door study in northern Tanzania. Epilepsia. 2009;50:2310-2313.

28. Serpa JA, Graviss EA, Kass JS, White AC Jr. Neurocysticercosis in Houston, Texas: an update. Medicine (Baltimore). 2011;90(1): $81-86$.

29. Sorvillo F, Wilkins P, Shafir S, Eberhard M. Public health implications of cysticercosis acquired in the United States. Emerg Infect Dis. 2011;17(1):1-6.

30. Medina MT, Aguilar-Estrada RL, Alvarez A, et al. Reduction in rate of epilepsy from neurocysticercosis by community interventions: the Salamá, Honduras study. Epilepsia. 2011;52(6):1177-1185.

31. Sotelo J, Diaz-Olavarrieta C. Neurocysticercosis: changes after 25 years of medical therapy. Arch Med Res. 2010;41(1):62-63.

32. Schantz PM, Moore AC, Muñoz JL, et al. Neurocysticercosis in an Orthodox Jewish community in New York City. $N$ Engl J Med. 1992;327(10):692-695.

33. Garcia HH, Del Brutto OH. Neurocysticercosis: updated concepts about an old disease. Lancet Neurol. 2005;4:653-661.

34. Estanol B, Corona T, Abad P. A prognostic classification of cerebral cysticercosis: therapeutic implications. J Neurol Neurosurg Psychiatr. 1986;49:1131-1134.

35. Carabin H, Ndimubanzi PC, Budke CM, et al. Clinical manifestations associated with neurocysticercosis: a systematic review. PLoS Negl Trop Dis. 2011;5:e1152.

36. Nash TE, Garcia HH, Rajshekhar V, Del Brutto OH. Clinical cysticercosis: diagnosis and treatment. In: Murrell KD, editor. WHO/FAO/OIE Guidelines for the Surveillance, Prevention and Control of Taeniosis/ Cysticercosis. Paris, France: OIE; 2005:73-99.

37. Nash TE, Mahanty S, Loeb JA, et al. Neurocysticercosis: a natural human model of epileptogenesis. Epilepsia. 2015;56(2):177-183. doi: 10.1111/epi.12849. 
38. Serpa JA, Yancey LS, White AC Jr. Advances in the diagnosis and management of neurocysticercosis. Expert Rev Anti Infect Ther. 2006;4:1051-1061.

39. Mishra D. Cysticercosis headache: an important differential of childhood headache disorder in endemic countries. Headache. 2007;47: 301-302.

40. Fogang YF, Camara M, Diop AG, Ndiaye MM. Cerebral neurocysticercosis mimicking or comorbid with episodic migraine? BMC Neurol. 2014; $14: 138$

41. Del Brutto OH, Del Brutto VJ. Calcified neurocysticercosis among patients with primary headache. Cephalalgia. 2011;32(3):250-254.

42. Cruz ME, Cruz I, Preux PM, Schantz P, Dumas M. Headache and cysticercosis in Ecuador, South America. Headache. 1995;35(2): 93-97.

43. Garcia HH, Nash TE, Del Brutto OH. Clinical symptoms, diagnosis, and treatment of neurocysticercosis. Lancet Neurol. 2014;13:1202-1215

44. Leon A, Saito EK, Mehta B, McMurtray AM. Calcified parenchymal central nervous system cysticercosis and clinical outcomes in epilepsy. Epilepsy Behav. 2015;43C:77-80.

45. Nash TE, Garcia HH. Diagnosis and treatment of neurocysticercosis. Nat Rev Neurol. 2011;7:584-594.

46. Winkler AS. Epilepsy and neurocysticercosis in sub-Saharan Africa. In: Foyaca-Sibat H, editor. Novel Aspects on Cysticercosis and Neurocysticercosis. Rijeka, Croatia: InTech; 2013:307-340.

47. Rangel R, Torres B, Del Brutto O, Sotelo J. Cysticercotic encephalitis: a severe form in young females. Am J Trop Med Hyg. 1987;36: 387-392.

48. Takayanagui OM, Odashima NS, Bonato PS, Lima JE, Lanchote VL. Medical management of neurocysticercosis. Expert Opin Pharmacother. 2011;12:2845-2856.

49. Pal DK, Carpio A, Sander JW. Neurocysticercosis and epilepsy in developing countries. J Neurol Neurosurg Psychiatr. 2000;68: 137-143.

50. Del Brutto OH, Rajshekhar V, White AC Jr, et al. Proposed diagnostic criteria for neurocysticercosis. Neurology. 2001;57:177-183.

51. Del Brutto OH, Santibanez R, Idrovo L, et al. Epilepsy and neurocysticercosis in Atahualpa: a door-to-door survey in rural coastal Ecuador. Epilepsia. 2005;46:583-587.

52. Nash TE, Del Brutto OH, Butman JA, et al. Calcific neurocysticercosis and epileptogenesis. Neurology. 2004;62:1934-1938.

53. Nash TE, Singh G, White AC, et al. Treatment of neurocysticercosis: current status and future research needs. Neurology. 2006;67: 1120-1127.

54. Tsang VC, Brand JA, Boyer AE. An enzyme-linked immunoelectrotransfer blot assay and glycoprotein antigens for diagnosing human cysticercosis (Taenia solium). J Infect Dis. 1989;159:50-59.

55. Rodriguez S, Dorny P, Tsang VC, et al. Detection of Taenia solium antigens and anti-T. solium antibodies in paired serum and cerebrospinal fluid samples from patients with intraparenchymal or extraparenchymal neurocysticercosis. J Infect Dis. 2009;199:1345-1352.

56. Del Brutto $\mathrm{OH}$. Diagnostic criteria for neurocysticercosis, revisited. Pathog Glob Health. 2012;106:299-304.

57. Machado Ldos R. The diagnosis of neurocysticercosis: a closed question? Arq Neuropsiquiatr. 2010;68:1-2.

58. Garcia HH, Gonzalez AE, Rodriguez S, et al. Neurocysticercosis: unraveling the nature of the single cysticercal granuloma. Neurology. 2010;75:654-658

59. Garcia HH, Evans CA, Nash TE, et al. Current consensus guidelines for treatment of neurocysticercosis. Clin Microbiol Rev. 2002;15: $747-756$

60. Garcia HH, Gonzalez AE, Gilman RH. Cysticercosis of the central nervous system: how should it be managed? Curr Opin Infect Dis. 2011;24:423-427.

61. Winkler AS, Willingham AL 3rd, Sikasunge CS, Schmutzhard E. Epilepsy and neurocysticercosis in sub-Saharan Africa. Wien Klin Wochenschr. 2009;121(Supp1 3):S3-S12.
62. Singh G, Rajshekhar V, Murthy JM, et al. A diagnostic and therapeutic scheme for a solitary cysticercus granuloma. Neurology. 2010;75: 2236-2245.

63. Gonzales I, Garcia HH. Current status and future perspectives on the medical treatment of neurocysticercosis. Pathog Glob Health. 2012;106:305-309.

64. Winkler AS. Neurocysticercosis in sub-Saharan Africa: a review of prevalence, clinical characteristics, diagnosis, and management. Pathog Glob Health. 2012;106:261-274.

65. Montano SM, Villaran MV, Ylquimiche L, et al. Neurocysticercosis: association between seizures, serology, and brain CT in rural Peru. Neurology. 2005;65:229-233.

66. Blocher J, Schmutzhard E, Wilkins PP, et al. A cross-sectional study of people with epilepsy and neurocysticercosis in Tanzania: clinical characteristics and diagnostic approaches. PLoS Negl Trop Dis. 2011;5:e1185

67. Takayanagui OM, Jardim E. Therapy for neurocysticercosis. Comparison between albendazole and praziquantel. Arch Neurol. 1992;49:290-294.

68. Garcia HH, Gilman RH, Horton J, et al. Albendazole therapy for neurocysticercosis: a prospective double-blind trial comparing 7 versus 14 days of treatment. Cysticercosis Working Group in Peru. Neurology. 1997;48:1421-1427.

69. Cardenas G, Carrillo-Mezo R, Jung H, Sciutto E, Hernandez JL, Fleury A. Subarachnoidal Neurocysticercosis non-responsive to cysticidal drugs: a case series. BMC Neurol. 2010;10:16.

70. Nash TE, Mahanty S, Garcia HH. Cysticercosis Group in Peru. Corticosteroid use in neurocysticercosis. Expert Rev Neurother. 2011;11:1175-1183.

71. Garcia HH, Gonzales I, Lescano AG, et al. Cysticercosis Working Group in Peru. Enhanced steroid dosing reduces seizures during antiparasitic treatment for cysticercosis and early after. Epilepsia. 2014;55:1452-1459

72. Mall RK, Agarwal A, Garg RK, Kar AW, Shukla R. Short course of prednisolone in Indian patients with solitary cysticercus granuloma and new onset seizures. Epilepsia. 2003;44:1397-1401.

73. Garg RK, Potluri N, Kar AM, et al. Short course of prednisolone in patients with cysticercus granuloma: a double blind placebo controlled trial. J Infect. 2006;53:65-69.

74. Prakash S, Garg RK, Kar AM, et al. Intravenous methylprednisolone in patients with solitary cysticercus granuloma: a random evaluation. Seizure. 2006;15:328-323.

75. Kishore D, Misra S. A short course of oral prednisolone on disappearance of lesion and seizure recurrence in patients of solitary cysticercus granuloma with single small enhancing CT lesion: an open label randomized prospective study. J Assoc Physicians India. 2007;55:419-424.

76. Keiser PB, Nash TE. Prolonged perilesional edema after treatment of parenchymal neurocysticercosis: methotrexate as a corticosteroidsparing agent. Clin Infect Dis. 2003;36:e122-e126.

77. Garcia HH, Pretell EJ, Gilman RH, et al. A trial of antiparasitic treatment to reduce the rate of seizures due to cerebral cysticercosis. $N$ Engl J Med. 2004;350:249-258.

78. Sotelo J, Del Brutto OH, Penagos P, et al. Comparison of therapeutic regimen of anticysticercal drugs for parenchymal brain cysticercosis. J Neurol. 1990;237:69-72.

79. Del Brutto OH, Roos KL, Coffey CS, Garcia HH. Meta-analysis: cysticidal drugs for neurocysticercosis: albendazole and praziquantel. Ann Intern Med. 2006;145:43-51.

80. Baird RA, Wiebe S, Zunt JR, Halperin JJ, Gronseth G, Roos KL. Evidence-based guideline: treatment of parenchymal neurocysticercosis: report of the guideline development subcommittee of the American academy of neurology. Neurology. 2013;80(15): 1424-1429

81. Castro N, Marquez-Caraveo C, Brundage RC, et al. Population pharmacokinetics of albendazole in patients with neurocysticercosis. Int $J$ Clin Pharmacol Ther. 2009;47:679-685. 
82. Gongora-Rivera F, Soto-Hernandez JL, Gonzalez Esquivel D, et al. Albendazole trial at 15 or $30 \mathrm{mg} / \mathrm{kg} /$ day for subarachnoid and intraventricular cysticercosis. Neurology. 2006;66:436-438.

83. Rajshekhar V. Albendazole therapy for persistent solitary cysticercus granulomas in patients with seizures. Neurology. 1993;43: $1238-1240$.

84. Thussu A, Chattopadhyay A, Sawhney IM, Khandelwal N. Albendazole therapy for single small enhancing CT lesions (SSECTL) in the brain in epilepsy. J Neurol Neurosurg Psychiatr. 2008;79: $272-275$.

85. Singh MK, Garg RK, Nath G, Verma DN, Misra S. Single small enhancing computed tomographic (CT) lesions in Indian patients with new-onset seizures. A prospective follow-up in 75 patients. Seizure. 2001;10:573-578.

86. Pretell EJ, Garcia HH, Custodio N, et al. Short regimen of praziquantel in the treatment of single brain enhancing lesions. Clin Neurol Neurosurg. 2000;102:215-218.

87. Medina-Santillan R, Mateos-Garcia E, Reyes-Garcia G, CastanedaHernandez G, Sotelo J. Pharmacoeconomic analysis of the short scheme praziquantel in the treatment of neurocysticercosis. Gac Med Mex. 2002;138:203-207.

88. Pretell EJ, Garcia HH, Gilman RH, Saavedra H, Martinez M. Cysticercosis Working Group in Peru. Failure of one-day praziquantel treatment in patients with multiple neurocysticercosis lesions. Clin Neurol Neurosurg. 2001;103:175-177.

89. Sotelo J, Jung H. Pharmacokinetic optimisation of the treatment of neurocysticercosis. Clin Pharmacokinet. 1998;34:503-515.

90. Carpio A, Santillan F, Leon P, Flores C, Hauser WA. Is the course of neurocysticercosis modified by treatment with anthelmintic agents? Arch Intern Med. 1995;155:1982-1988.

91. Maiga Y, Diallo M, Bouteille B, et al. About an autochtonous case of neurocysticercosis in Mali. Bull Soc Pathol Exot. 2009;102: 211-214.

92. Sotelo J, Marin C. Hydrocephalus secondary to cysticercotic arachnoiditis. A long-term follow-up review of 92 cases. J Neurosurg. 1987;66:686-689.

93. Suastegui Roman RA, Soto-Hernandez JL, Sotelo J. Effects of prednisone on ventriculoperitoneal shunt function in hydrocephalus secondary to cysticercosis: a preliminary study. J Neurosurg. 1996;84:629-633.
94. Gabriel S, Blocher J, Dorny P, et al. Added value of antigen ELISA in the diagnosis of neurocysticercosis in resource poor settings. PLoS Negl Trop Dis. 2012;6:e1851.

95. Schantz PM, Cruz M, Sarti E, Pawlowski Z. Potential eradicability of taeniasis and cysticercosis. Bull Pan Am Health Organ. 1993;27: 397-403.

96. Del Brutto OH. Neurocysticercosis. Continuum Lifelong Learn Neurol. 2012;18(6):1392-1416.

97. Del Brutto OH, Garcia HH. Cysticercosis of the Human Nervous System. Berlin, Germany; Berlin Heidelberg: Springer; 2014.

98. Pondja A, Neves L, Mlangwa J, et al. Use of oxfendazole to control porcine cysticercosis in a high-endemic area of Mozambique. PLoS Med. 2012;6(5):1-5.

99. Nascimento E, Costa JO, Guimaraes MP, Tavares CA. Effective immune protection of pigs against cysticercosis. Vet Immunol Immunopathol. 1995;45(1-2):127-137.

100. Plancarte A, Flisser A, Gauci CG, Lightowlers MW. Vaccination against Taenia solium cysticercosis in pigs using native and recombinant oncosphere antigens. Int J Parasitol. 1999;29(4): 643-647.

101. Verastegui M, Gilman RH, Gonzalez AE, et al. Taenia solium oncosphere antigen induce immunity in pigs against experimental cysticercosis. Vet Parasitol. 2002;108:49-62.

102. Flisser A, Gauci CG, Zoli A, et al. Induction of protection against porcine cysticercosis by vaccination with recombinant oncosphere antigens. Infect Immun. 2004;72(9):5292-5297.

103. Gonzalez AE, Gauci CG, Barber D, et al. Vaccination of pigs to control human neurocysticercosis. Am J Trop Med Hyg. 2005;72(6): 837-839.

104. Assana E, Kyngdon CT, Gauci CG, et al. Elimination of Taenia solium transmission to pigs in a field trial of the TSOL18 vaccine in Cameroon. Int J Parasitol. 2002;40(5):515-519.

105. Centers for Disease Control and Prevention. Cysticercosis. Atlanta, GA: Centers for disease control and prevention. Available from: http://www. cdc.gov/parasites/cysticercosis/biology.html. Accessed July 6, 2015.
International Journal of General Medicine

\section{Publish your work in this journal}

The International Journal of General Medicine is an international, peer-reviewed open-access journal that focuses on general and internal medicine, pathogenesis, epidemiology, diagnosis, monitoring and treatment protocols. The journal is characterized by the rapid reporting of reviews, original research and clinical studies across all disease areas.

\section{Dovepress}

A key focus is the elucidation of disease processes and management protocols resulting in improved outcomes for the patient. The manuscript management system is completely online and includes a very quick and fair peer-review system. Visit http://www.dovepress.com/ testimonials.php to read real quotes from published authors. 